ISSN 1518-3483

Licenciado sob uma Licença Creative Commons

\title{
Desenvolvimento de expertise: um estudo de caso ${ }^{1}$
}

\section{Expertise development: a case study}

\begin{abstract}
Afonso Galvão ${ }^{[a]}$, Cátia Perfeito ${ }^{[b]}$, Ricardo Macedo ${ }^{[c]}$
[a] Ph.D. pela University of Reading, Inglaterra, professor do Programa de Doutorado em Educação e dos Programas de Mestrado em Educação e Psicologia da Universidade Católica de Brasília, Brasília, DF Brasil, e-mail: agalvao@ucb.br

[b] Mestranda do Programa de Mestrado em Educação da Universidade Católica de Brasília, professora do ensino fundamental da Secretaria de Estado de Educação do Distrito Federal, Brasília, DF - Brasil, e-mail: catiaperfeito@yahoo.com.br

[c] Mestrando do Programa de Mestrado em Educação da Universidade Católica de Brasília, funcionário do Banco do Brasil, Brasília, DF - Brasil, e-mail: rm.macedo@ig.com.br
\end{abstract}

\section{Resumo}

Nesta pesquisa qualitativa de caráter exploratório, objetivou-se investigar as trajetórias de aprendizagem que levaram um indivíduo a se tornar expert em uma área de conhecimento, à luz das teorias sobre expertise. Buscou-se pesquisar se o processo de desenvolvimento

${ }^{1}$ Pesquisa financiada pelo CNPq. 
vivenciado coincidia com os fatores apresentados na literatura como determinantes para o alcance da expertise, tais como: condições ambientais favoráveis, estudo individual deliberado, personalidade e aspectos motivacionais. O instrumento metodológico utilizado foi a entrevista semiestruturada. Foi entrevistado um expert, cujo domínio de expertise é a avaliação da aprendizagem. Os resultados sugerem que o participante contou com um ambiente estimulador desde a infância e apresentou forte motivação pessoal para permanecer na área por longo prazo. Aspecto diverso ao apresentado na literatura, que emergiu na pesquisa, diz respeito aos momentos iniciais do desenvolvimento da expertise, em que há indicações de que críticas desfavoráveis, na verdade, estimularam o envolvimento com a área.

Palavras-chave: Desenvolvimento. Expertise. Estudo deliberado.

\section{Abstract}

This qualitative exploratory research aimed at investigating the ways in which a person became an expert at a field of knowledge, taking into account expertise theory. The study focused on the participant's process of professional development and check whether this coincided with those factors the literature point out as determinants for expertise development such as favorable environmental conditions, deliberate practice, personality and motivation. An expert whose domain of knowledge is learning evaluation was interviewed at depth by means of semi structured interview. Results suggest that the participant had, since childhood, an stimulating environment and presented a strong motivation to stay in his area of expertise in the long term. However, rather differently from a dominant view in the literature, it emerged from this research that in the first moments of involvement with the field there were several severe criticism on his performance which actually served as motivational factors to get himself deeply involved with the area.

Keywords: Development. Expertise. Deliberate practice.

\section{Introdução}

Os primeiros estudos acerca da expertise coincidem com a história da pesquisa experimental psicológica, todavia tais estudos ocupavam-se 
mais em descrever fases de desenvolvimento de uma habilidade específica do que em oferecer teorias que justificassem aspectos comuns da expertise em campos distintos (GALVÃO, 2003). Outras pesquisas apoiavam a ideia de que a habilidade expert estaria vinculada a causas inatas, desse modo a performance superior de determinado indivíduo estava vinculada à habilidade natural herdada (GALTON, 1979). Foi a partir de um artigo de Simon e Chase (1973) que uma teoria geral para a estrutura da expertise foi proposta. Essa teoria foi capaz de oferecer as primeiras previsões empíricas para o desempenho expert em uma variedade de domínios, destacando música, medicina, xadrez e outros. Pesquisas sob esse enfoque ofereceram indícios fortes de que o alcance da expertise resultaria de uma trajetória de desenvolvimento individual que envolvia, especialmente, uma dedicação intensiva ao domínio escolhido.

Apesar dos significativos avanços rumo à compreensão da $e x-$ pertise, ainda persiste, na literatura, a ausência de uma definição sistemática que seja compartilhada por diferentes pesquisadores. Assim, buscou-se em French e Sternberg (1989, p. 158) uma referência à expertise, compreendendo-a como uma "capacidade, adquirida pela prática, de desempenhar qualitativamente bem uma tarefa particular de um domínio". A definição proposta apresenta três fatores importantes para o tratamento do tema: noção de prática, também entendida como estudo deliberado, qualidade do desempenho e noção de domínio específico (GALVÃO, 2003).

O estudo individual deliberado é tido como um dos mais importantes fatores relacionados ao desenvolvimento da expertise. Segundo Galvão e Galvão (2006), o estudo deliberado é uma atividade em que o aprendiz é capaz de controlar e tomar as iniciativas do processo, isto é, uma atividade cuja finalidade é a melhoria do desempenho. Nesse sentido, tornar-se um expert exige o envolvimento do sujeito em atividades praticadas regularmente de modo deliberado, qualitativa e quantitativamente eficiente, por longo prazo. Alguns pesquisadores (GALVÃO, 2003; FRENCH; STERNBERG, 1989) sugerem que o conhecimento expert é consequência de pelo menos dez anos de estudo deliberado em um domínio específico. 
O segundo fator mencionado na definição refere-se à qualidade do desempenho. De acordo com Galvão (2003), existem duas considerações a fazer sobre esse aspecto, que percorrem caminhos distintos, mas inter-relacionados, que levam em conta a definição de expertise e de inteligência e a análise da qualidade de desempenho como conceito construído. Primeiramente, expertise e inteligência são definidas a partir de diferenças individuais, uma vez que o que diferencia experts de aprendizes é a capacidade superior de resolução de problemas dos primeiros. Em decorrência, estudiosos concebem os experts como um caso de raciocínio inteligente especial (FRENCH; STERNBERG, 1989) ou entendem expertise e inteligência como termos sinônimos (CECI; LIKER, 1986). Soma-se a isso a ideia de que a qualidade de desempenho do sujeito envolve um referencial social construído, isto é, o reconhecimento de uma dada habilidade expert dependerá grandemente da cultura em que está inserida, influenciando a forma como as pessoas envolvidas se dedicam à área de domínio (GALVÃO, 2003).

A terceira implicação importante desenhada na definição da expertise diz respeito ao domínio específico. Para French e Sternberg (1989), a dedicação a uma área específica leva à compreensão da expertise como uma habilidade adquirida por meio da prática, em lugar de, por exemplo, uma habilidade herdada. Esse ponto de vista não reforça, de maneira alguma, que um indivíduo não possa ser altamente habilidoso em mais de um domínio ou que a expertise em uma área seja consequência de expertise em outro domínio.

O estudo deliberado é particularmente relevante nas pesquisas sobre os processos de aprendizagem expert. Todavia, considerá-lo o fator mais importante representa um exagero, pois a prática deliberada interage com outros fatores, tais como características cognitivas do aprendiz, personalidade, condições ambientais, diferenças nas primeiras experiências de vida, preferências, hábitos, oportunidades, resiliência, aspectos motivacionais, envolvimento emocional com o produto a ser apreendido, entre outros, para que a expertise seja atingida (GALVÃO, 2003, 2007b; GALVÃO; GALVÃO, 2006).

As pesquisas sobre o desenvolvimento da expertise não se ocuparam meramente em identificar e descrever o comportamento expert, mas também 
em compreender como o conhecimento é adquirido (ERICSSON; SMITH, 1994) e como esse conhecimento é organizado ou estruturado (BEDARD; CHI, 1992). No momento, o conhecimento produzido acerca da expertise tem sido utilizado com o objetivo de fazer com que mais pessoas possam atingir níveis altos de competência em diferentes áreas (CHI, 2006). De acordo com Galvão (2003), não há indícios de que o desenvolvimento da expertise seja restrito a um grupo específico de indivíduos ungidos desde o nascimento com qualidades especiais diferenciadoras; ao contrário, quanto mais se investiga a área, mais forte é a convicção de que qualquer pessoa com desenvolvimento normal pode se tornar altamente habilidosa em um domínio.

O conhecimento sobre o desenvolvimento da habilidade expert tem sido tradicionalmente pesquisado com uso da metodologia de estudo de caso. No Brasil, poucas investigações têm focalizado esse tema. Nesse contexto, esta pesquisa objetivou explorar aspectos das primeiras experiências de aprendizagem de um expert. Particularmente, foram focalizadas as origens do interesse pela área, processos de estudo deliberado, autorregulação, aspectos motivacionais, considerando o contexto familiar. Em outras palavras, investigou-se de modo sistêmico um caso de aprendizagem expert.

\section{Metodologia}

Nesta pesquisa qualitativa de caráter exploratório, utilizou-se a entrevista semiestruturada como instrumento de coleta de dados. O roteiro da entrevista foi estruturado a partir de sete questões geradoras, as quais se relacionavam aos objetivos do estudo. O caso objeto deste estudo teve como participante um expert do sexo masculino, professor de pós-graduação, cujo domínio de expertise é "avaliação da aprendizagem". O critério utilizado para a seleção do participante foi seu reconhecimento profissional no meio acadêmico em que atua e a indicação de pares e ex-alunos. A entrevista, que foi gravada, ocorreu na sala do participante e teve duração de aproximadamente uma hora. Complementaram os dados, 15 sessões de observação participante das aulas do professor. 
Para análise dos dados, utilizou-se a estratégia da análise de discurso (BRANDÃO, 2002; ORLANDI, 2002). Os dados foram transcritos na íntegra e alocados a grandes categorias, definidas a priori a partir dos objetivos específicos. Em seguida, subcategorias foram criadas em correspondências aos conteúdos discursivos específicos. Segmentos de fala foram, então, justapostos por critérios associativos de contingência, contraste ou similaridade.

\section{Resultados e discussão}

\section{Primeiras experiências de vida e talento}

Existe, na sociedade e em parte da literatura psicológica, um reconhecimento de que o indivíduo, para alcançar um desempenho expert em uma determinada área, necessitaria possuir desde muito jovem algo especial que o diferenciaria e facilitaria o desenvolvimento da expertise. Pesquisas sobre crianças prodígio revelaram que elas demonstram suas habilidades precocemente (GALVÃO, 2003); entretanto outros estudos, retrospectivos, sobre o processo inicial de aprendizagem de experts indicaram que eles não eram promissores quando iniciantes (GALVÃO, 2003; SOSNIAK, 1985). Considerando essas indicações, buscou-se investigar se o entrevistado era considerado pelas pessoas próximas uma criança que se destacava em alguma área específica. O discurso do entrevistado revelou uma aproximação aos estudos retrospectivos citados por Galvão (2003): "eu era considerado mais comportado que talentoso. Eu era um menino de muito movimento. Mas, eu dava trabalho para os meus pais".

Por outro lado, o tema talento é um dos mais controversos na literatura psicológica, pois ainda persistem dificuldades em defini-lo, estruturá-lo e aplicá-lo (GALVÃO, 2006); todavia um dos aspectos importantes relacionados ao tema refere-se a suas características inatas. As primeiras pesquisas sobre expertise apoiavam a visão de que ela, em diversos domínios, resultaria de causas inatas, em consequência do fato 
de que sujeitos eminentes tinham filhos eminentes (GALTON, 1979). Tal concepção foi observada na fala do entrevistado ao declarar que talento refere-se a um desempenho acima da média, com surgimento precoce. Algo que o participante não acreditava possuir.

Para mim, uma pessoa talentosa revela... É uma pessoa que se desempenha excepcionalmente em alguma coisa, desde a psicomotricidade, executando bem um instrumento, jogando bem um esporte. Excepcionalmente, acima da média. Acima da média, por exemplo, uma criança que aos sete anos consegue tocar uma valsa de Chopin ao piano, para mim ela é uma pessoa talentosa.

Percebe-se que a noção de talento entendida pelo entrevistado compreende uma série de variáveis que vão desde o senso comum até as questões de altas habilidades. De acordo com o senso comum, a noção de talento é frequentemente associada à habilidade individual que permite a um sujeito desempenhar muito bem uma determinada área sem a necessidade de prática intensiva (GALVÃO, 2004). Concebe-se que o aprendizado para as pessoas talentosas ocorre de forma mais fácil e com menos esforço. Por outro lado, a pessoa tida como não talentosa é aquela que precisará dedicar o dobro do tempo em uma atividade, quando comparada ao talentoso. No entanto, Galvão (2007a) destaca que não existe uma maneira de identificar talento como resultado maior de habilidade.

Eu sou uma pessoa esforçada. Não me considero uma pessoa talentosa. Eu me considero uma pessoa que sua, que mete as mãos na parede para poder subir. [...] Acontece com a pessoa como eu de chegar longe, tanto quanto ou mais do que um talentoso. [...] Em grande parte dos casos, porque o talentoso não é suficientemente desafiado.

As explicações acerca do desenvolvimento de experts têm sido oferecidas no sentido de compreender melhor esse processo, sendo, por vezes, conflituosas. As pesquisas sobre a expertise musical têm ajudado a lançar luz sobre algumas questões. Pesquisas de Sosniak $(1985,1990)$ com pianistas novatos considerados talentosos, por exemplo, revelaram que não havia garantia de que seriam músicos experts quando adultos. 
O progresso desses músicos foi compreendido como uma combinação de ambiente adequado e uma rede de apoio social que valorizava seus esforços, especialmente os pais. Nesse sentido, as primeiras experiências de vida do novato revelam um processo de aprendizagem monitorado pelos pais, professores e outros que lhe dão suporte para que se mantenham no processo de aperfeiçoamento.

Além do episódio que tive com minha professora de Medidas no mestrado, eu tive um episódio no ensino médio em História. [...] Eu comecei a vagabundear na disciplina dele. [...] E mandou-me a caneta em cima e me deu um, dois, sei lá o quê. [...] Meu pai me disse: cara! Você não está estudando! Você está faltando aula... Você me desculpe. Aí, eu reagi.

Corroborando outros estudos (GALVÃO, 2003, 2007a; HUNT, 2006; SOSNIAK, 1990), percebe-se que a questão do talento não é preponderante para o alcance da expertise em algum domínio específico, como pensam os leigos, pois envolve, além do comprometimento dos familiares, outros aspectos, como estudo individual deliberado, ambiente e outros, que atuam em grau e intensidade distintos, porém de maneira interativa.

\section{Momentos iniciais do interesse pela área}

No que diz respeito aos momentos iniciais do desenvolvimento da expertise do entrevistado, há indicações de uma estrutura psíquica que se sentiu motivada pela crítica de um professor. O participante declarou que foi o insucesso ao ser avaliado que o levou ao interesse por avaliação. Um conjunto de fatores que costuma desmotivar outras pessoas, por mexer com o narcisismo e com a autoestima, aqui se revelou estimulador. A fala abaixo ilustra a análise:

[...] como comecei a me interessar? Pelo insucesso. [...] No meu mestrado, eu fiz uma disciplina, Medidas de Avaliação, e tive a menor nota de todas as disciplinas que eu cursei no mestrado. [...] Mas aí, eu processei isso com 
muita lentidão. Estive chateado, mas eu vi que não podia ficar lambendo minhas feridas com esse negócio e resolvi agarrar o touro pelo chifre e ir estudar. E então, sozinho, depois do insucesso, eu fui estudar.

De acordo com Fontana (1991), o baixo desempenho escolar produz uma baixa autoestima ou a rejeição pela escola, que passa a ser considerada entediante. $\mathrm{O}$ insucesso conduz o aluno, às vezes, a acreditar que não será capaz de superar as dificuldades. Todavia, o discurso analisado revela um movimento contrário ao encontrado na literatura. Observase um indivíduo que, desde a juventude, sentia-se estimulado por uma avaliação desfavorável.

[...] Eu fui estudioso quando fui desafiado. [...] Desafiado é julgado ruim, desfavoravelmente. Além desse episódio que eu tive com a professora de Medidas no mestrado, eu tive um episódio no ensino médio em História, com o professor M. J. Eu comecei a vagabundear na disciplina dele. [...] E mandou-me a caneta em cima e me deu um teste, me deu um, dois [...] Aí eu comecei a achar ruim, espernear e reclamar. [...] Fui estudar, não com o texto dele, fui pegar um livro mais embaixo. E saí-me bem. Veja bem! Eu preciso ser desafiado, dizendo você não sabe, não sabe bem, está insatisfatório.

Embora seja normal que o insucesso leve à desmotivação, alguns autores sustentam que estudantes com boa autoestima tendem a oferecer explicações transitórias para fracassos localizados, tese presente na teoria da atribuição (WEINER, 1974) e apoiada por outras pesquisas (GALVÃO, 2000; EGREJA, 2007).

\section{Estudo individual deliberado}

Estudos sobre expertise têm demonstrado que o indivíduo se torna altamente habilidoso em um determinado domínio por meio de atividades regulares praticadas de modo deliberado. Assim, tornar-se um expert requer o envolvimento do indivíduo com uma área específica por 
longo prazo, ou seja, exige pelos menos dez anos de estudo individual deliberado, qualitativamente e quantitativamente eficiente, para que a expertise seja atingida (GALVÃO, 2003). A prática intensiva para atingir a expertise emergiu no contexto da entrevista. O participante relatou que dedicou muitos anos ao domínio da avaliação, após o episódio que considerou como marco do início do interesse pela área.

Eu vou tomar como marco aquele mau episódio. Eu cursei aquela disciplina, acredito que foi em [mil novecentos e] setenta e oito. Então, [mil novecentos e] noventa e oito deu vinte anos. Estamos há quase trinta anos, que eu venho estudando. [...] A minha área de expertise, aí sim eu sou professor. [...] Aí, uma vez você identificando esse território em que você é singular, investir: estudar bastante, escrever, pesquisar, debater com pessoas que são do mesmo nivel de interesse, enfim.

Todavia, não surgiu nas falas do entrevistado elementos que sugerissem a dedicação intensiva como maçante. O que vem corroborar o resultado dos estudos de Galvão e Lacorte (2007), que desmistificam o estudo individual deliberado como uma coisa "chata" e "desprazerosa". Galvão e Lacorte (2007), ao investigar como músicos populares aprendem o ofício, revelaram que eles estudam muito, isto é, dedicam-se deliberadamente ao aprendizado da profissão, mas nem sempre de forma homogênea e convencional. A esse respeito, encontraram-se, nesta pesquisa, indícios de uma prática de estudo que corresponde aos achados dos pesquisadores citados. $\mathrm{O}$ participante, ao ser perguntado sobre o tempo dedicado ao estudo do domínio, o descreveu como inconstante e que atendia às exigências da profissão.

[...] Não era muito constante. Eu lhe digo que houve lapsos de investimento. Presentemente, por exemplo, se você me perguntar: esta semana, na semana passada, quanto você investiu no estudo de avaliação?[...] Eu trabalhei duas horas. Então, duas horas, mas eu estou numa fase que estudar e trabalhar com avaliação está sendo uma coisa espasmódica. Em contra posição a isso, por exemplo, quando eu lecionei a disciplina "avaliação", o meu investimento era mais continuado, mais constante. Então, é dependendo do período, o meu investimento é mais concentrado ou mais ralo. 
Para Galvão (2007b), o estudo individual deliberado é tido como um fator importante para o desenvolvimento da expertise em qualquer domínio. A prática deliberada envolve boa metacognição, isto é, capacidade para organizar o próprio conhecimento e conseguir superar limitações.

\section{Autorregulação}

Autorregulação é um importante aspecto do processo de aprendizagem e uma função cognitiva que opera na base de sua ocorrência. Refere-se aos mecanismos que as pessoas usam para controlar o seu próprio processo de aprendizagem. Implica estabelecer um objetivo de estudo e controlar o próprio progresso, utilizando estratégias, tais como monitoração, elaboração e gerenciamento de esforço (CORNO, 1989). Essas estratégias determinam decisões posteriores sobre continuar ou não alocando tempo para o estudo, bem como mudanças nas estratégias de aprendizagem (THIEDE; DUNLOSKY, 1999). Em geral, estudantes podem ser descritos como aprendizes autorregulados, em razão da ativa participação que têm em seu próprio processo de aprendizagem em termos de metacognição, comportamento e motivação (GALVÃO, 2003).

Para Gomes (2008), a obrigatoriedade de aprendizagem, inicialmente, se coloca a serviço de pais, professores e outros que lhes dão o feedback necessário para manter-se nas relações de educação. Depois, a pressão externa e a vontade interna vão se autorregulando diante do campo de conhecimento que lhes gratifica e, assim, dá o contorno particularizado à sua área de interesse, internalizando pressões e necessidades que antes provavelmente se configurassem como predominantemente externas. É o que podemos constatar na fala do entrevistado:

[...] Se você disser: olha! Não está bom isso aqui de jeito nenhum. Eu vou escutar e vou tentar voltar para a prancheta e modificar. Se você me disser catorze vezes: corrija isso, corrija isso, corrija isso... E eu, muito provavelmente, vou voltar e refazer. 


\section{Ambiente de aprendizagem e motivação}

Segundo Campos (1997), a aprendizagem envolve o uso e o desenvolvimento de todos os poderes, capacidades, potencialidades da pessoa, tanto físicas quanto mentais e afetivas. Isso significa que a aprendizagem não pode ser considerada somente como um processo de memorização ou que emprega apenas o conjunto de funções mentais ou unicamente os elementos físicos ou emocionais, pois todos esses aspectos são necessários.

A partir desses cenários, e adotando como premissa que muitas são as características do ambiente de aprendizagem, buscou-se compreender a extensão e profundidade desse ambiente em suas diversas dimensões, como, por exemplo: ambiente sociocultural, ambiente físico, rede de apoio social (familiares, professores e amigos), oportunidades (condições financeiras), motivação, entre outros.

Para Galvão (2003), há uma tendência entre pesquisadores em expertise de enfatizar a capacidade do expert como aprendida, isto é, como consequência de condições adequadas de aprendizagem sustentadas por longo prazo. Os diversos estudos revelam que uma das dimensões que mais se destacam no ambiente de aprendizagem diz respeito ao ambiente sociocultural, onde o indivíduo viveu suas primeiras experiências na infância, bem como os traços de personalidade ali constituídos (ALENCAR; GALVÃO, 2007). Outra dimensão de fundamental importância é o apoio parental, pois em um ambiente familiar onde os pais encorajam, incentivam e apoiam o interesse específico dos seus filhos os resultados são significativamente superiores (SOSNIAK, 1990). Pode-se constar a efetividade dessas dimensões a partir das falas do entrevistado:

eu tinha bons exemplos em casa, bons modelos. Meu pai era professor, um bom professor. A minha mãe também. Minha madrasta foi uma boa professora. Eles me estimulavam sendo bons professores. Eu tinha bons modelos em casa. Eu tinha muitos livros e modelos. 
Outra característica importante do aprendizado expert diz respeito ao intenso envolvimento no trabalho realizado (ALENCAR; GALVÃO, 2007). Geralmente, os psicólogos utilizam o termo motivação para fazer referência ao movimento que leva o indivíduo a se dedicar intensamente à produção de um trabalho. A motivação é um construto que envolve diferentes variáveis, de forma que, até o momento, não há um consenso quanto à sua definição. Todavia, constata-se, em grande parte das definições de motivação, a ênfase dada à escolha de uma ação e ao esforço e à persistência para sua concretização (RAVANELLO, 2008).

De acordo com Cratty (1984), a motivação é conceituada como o processo que leva as pessoas a uma ação ou inércia em diversas situações, o qual, ainda, pode ser o exame das razões pelas quais se escolhe fazer algo e executar algumas tarefas com maior empenho do que outras. Por sua vez, Magill (1984) se refere à motivação como causa de um comportamento, pois define motivação como alguma força interior, impulso ou uma intenção, que leva uma pessoa a fazer algo ou agir de certa forma. Os trechos da fala a seguir servem como evidência:

como eu comecei a me interessar? Pelo insucesso. No meu mestrado, eu fiz uma disciplina, Medidas e Avaliação, e tiver a menor nota de todas as disciplinas que eu cursei no mestrado. [...] Eu acho que se eu tivesse tirado um "A" nesta disciplina, provavelmente eu não fosse instigado a estudá-la melhor.

Contudo, segundo Fontana (1991), por conveniência pode-se dividi-las em formas intrínsecas de motivação, que provêm do indivíduo, e extrínsecas, que lhe são impostas pelo ambiente. Considerando as falas do entrevistado, pode-se afirmar que ele é partidário da proposta de Fontana:

no meu caso, a motivação tem que ser fundamentalmente intrínseca. Se você me perguntar, pegando o contínuo "motivação", num pólo botando intrínseca e extrínseca; eu aloco oito, de zero a dez, aqui e dois na extrínseca. Eu me sinto motivado quando eu consigo um resultado de um trabalho pessoal meu. 
Todavia, também é perfeitamente perceptível a forte influência de fatores motivacionais extrínsecos, como, por exemplo: a família, as condições financeiras que possibilitaram a oportunidade de estudar no exterior, a professora, muitos livros, etc.

Eu tinha bons exemplos em casa, bons modelos. Meu pai era professor, um bom professor. A minha mãe também. Minha madrasta foi uma boa professora. Eles me estimulavam sendo bons professores. Eu tinha bons modelos em casa. Eu tinha muitos livros e modelos.

Sim! [fiz mestrado fora do país] Eu fiz em educação. [...] Então, eu fiz mestrado e, logo em seguida, o doutorado. Foi consecutivo.

\section{Personalidade}

Ainda tratando das dimensões do ambiente de aprendizagem, vale a pena destacar a personalidade do aprendiz e, no caso específico do nosso estudo, os traços de autoestima, a introversão e a extroversão. Campos (1997) apresenta algumas características da aprendizagem, enfatizando que é um processo que envolve mudança de comportamento do indivíduo, o qual utiliza todos os aspectos constitutivos de sua personalidade no ato de aprender, a fim de que se restabeleça o equilíbrio vital, rompido com o aparecimento da situação-problema.

[...] como comecei a me interessar? Pelo insucesso. Na minha... No meu mestrado, eu fiz uma disciplina, Medidas de Avaliação, e tive a menor nota de todas as disciplinas que eu cursei no mestrado. [...] Mas aí eu processei isso com muita lentidão. Estive chateado com isso, mas resolvi, eu vi que não podia ficar lambendo minhas feridas com esse negócio e resolvi agarrar o touro pelo chifre e ir estudar. E então, sozinho, depois do insucesso, eu fui estudar.

As modificações de comportamento decorrentes da experiência podem levar a frustrações e perturbações emocionais, quando não se dá integração do comportamento. Quando, na realidade, a aprendizagem se realiza, surge um novo comportamento, capaz de solucionar a situação 
problemática encontrada, levando o aprendiz à adaptação ou à integração de sua personalidade ou ao ajustamento social. A acumulação das experiências leva à organização de novos padrões de comportamento, que são adquiridos pelo sujeito. Daí se afirmar que quem aprende modifica seu comportamento (CAMPOS, 1997).

Eu ligo esforço à perseverança. E perseverança que eu associo à palavra grega que define a capacidade de você retomar a trilha depois do insucesso. Só há perseverança quando você erra, quando você não se deu bem, quando você fracassou, em outras palavras.

\section{Conclusões}

A trajetória de aprendizagem do participante condiz com os aspectos apresentados na literatura como fundamentais para o alcance da expertise. O participante envolveu-se no estudo intensivo da área por cerca de 30 anos, apresentando, todavia, momentos de pouca dedicação ao domínio, semelhante ao processo de aprendizagem de músicos populares descrito por Galvão e Lacorte (2007).

A fala do entrevistado revelou que ele não era considerado um menino talentoso. Todavia, não há como identificar o termo talento como causa de maior habilidade. Por outro lado, o participante contou com uma rede de apoio social, especialmente os pais, que o estimularam a estudar, serviram como modelo e proporcionaram os recursos necessários (GALVÃO, 2007a).

Aspecto diverso ao apresentado na literatura, que surgiu na pesquisa, diz respeito aos momentos iniciais do desenvolvimento da expertise, em que há indicações de uma estrutura psíquica motivada por críticas desfavoráveis ou pelo insucesso. Pesquisa de Frakes (1984) relacionou o desejo de continuar atividades de um domínio específico com a autopercepção positiva de habilidade, identificando a falta de reconhecimento de habilidade a caminhos que levam à frustração e à desistência. Todavia, os achados desta pesquisa revelaram que um conjunto de fatores que 
costuma desmotivar outros indivíduos, por abalar a autoestima, revelou-se, aqui, estimulador.

Outro ponto importante a considerar refere-se à ratificação de que o estudo individual deliberado é apenas um dos fatores que contribuem para o alcance da expertise. Dessa forma, identificar o processo de aprendizagem expert com uma função monotônica representa um reducionismo, que opera muito próximo da noção de talento (GALVÃO, 2007a). A aprendizagem e o desempenho humano são resultado de muitos fatores interativos, como os apresentados nesta pesquisa, a saber: diferenças nas primeiras experiências de vida, rede social de apoio, aspectos motivacionais, características de personalidade, envolvimento emocional com o produto a ser apreendido e outros (GALVÃO, 2003, 2006, 2007b; GALVÃO; GALVÃO, 2006). E sobre esse último ainda há um amplo espectro de possibilidades de investigação, uma vez que a interação entre emoção e cognição até o momento não foi suficientemente explorada (GALVÃO, 2003).

Embora os estudos sobre a expertise tenham avançado rumo à compreensão desse fenômeno, as trajetórias de desenvolvimento dos experts ainda precisam ser mais examinadas, considerando o papel da escola, dos professores e de pessoas significativas, se as estratégias de estudo de uma área podem ser transferidas para outra e as razões que levaram a eleger determinado domínio. Em verdade, o objetivo almejado é entender como experts tornam-se o que são para que outros possam se tornar mais habilidosos.

\section{Referências}

ALENCAR, E. M. L. S. de; GALVÃO, A. C. T. Condições favoráveis à criação nas ciências e nas artes. In: VIRGOLIM, Â. M. R. (Org.). Talento criativo: expressão em múltiplos contextos. Brasília: Editora UnB, 2007. v. 1, p. 103-120.

BEDARD, J.; CHI, M. T. H. Expertise. Current Directions in Psychological Science, v. 1, p. 135-139, 1992. 
BRANDÃO, H. H. N. Introdução à análise do discurso. Campinas: Ed. da Unicamp, 2002.

CAMPOS, D. M. de S. Psicologia da aprendizagem. Petrópolis: Vozes, 1997. CECI, S.; LIKER, J. A day at the races: a study of IQ, expertise, and cognitive complexity. Journal of Experimental Psychology: General, p. 255-266, 1986.

CHI, M. T. H. Two approaches to the study of experts'characteristics. In: ERICSSON, K. A. et al. (Ed.). The Cambridge handbook of expertise and expert performance. Cambridge; New York: Cambridge University Press, 2006. p. 21-30.

CORNO, L. Self-regulated learning: a volitional analysis. In: ZIMMERMAN, B.; SCHUNK, D. Self-regulated learning and academic achievement: theory, research and practice. New York: Springer, 1989. p. 111-141.

CRATTY, B. J. Psicologia do esporte. 2. ed. Rio de Janeiro: Prentice Hall do Brasil, 1984.

EGREJA, J. J. C. Representações sociais do sucesso acadêmico na perspectiva de alunos bem-sucedidos. 2007. 88 f. Dissertação (Mestrado em Educação) - Universidade Católica de Brasília, Brasília, 2007.

ERICSSON, K. A.; SMITH, J. Prospects and limits of empirical study of expertise: an introduction. In: ERICSSON, K. A.; SMITH, J. Toward a general theory of expertise: prospects and limits. Cambridge: Cambridge University Press, 1994. p. 1-38.

FONTANA, D. Psicologia para professores. 2. ed. São Paulo: Manole, 1991.

FRAKES, L. Differences in music achievement, academic achievement, and attitude among participants, dropouts and nonparticipants in secondary school music. 1984. 370 f. Thesis (Ph.D. Education) - University of Iowa, Iowa City, 1984.

FRENCH, P. A.; STERNBERG, R. J. Expertise and intelligent thinking: when is it worse to know better? In: STERNBERG, R. J. Advances in the psychology of human intelligence. New Jersey: Lawrence Erlbaum Associates, 1989. v. 5, p. 157-188. 
GALTON, F. Hereditary genius: an inquiry into its laws and consequences. London: Julian Friedman, 1979.

GALVÃO, A. C. T. Practice in orchestral life: an exploratory study of string players learning processes. 2000. 400 f. Thesis (Ph.D. Education) - Reading University, UK, 2000.

GALVÃO, A. C. T. Pesquisa sobre expertise: perspectivas e limitações. Temas em Psicologia, v. 9, n. 3, p. 53-68, 2003.

GALVÃO, A. C. T. Teachers' social representation of talent in Brasilia. In: CONFERENCE OF THE EUROPEAN COUNCIL FOR HIGH ABILITY, 9., 2004, Pamplona. Proceedings... Pamplona: University of Pamplona Press, 2004. p. 1-15.

GALVÃO, A. C. T.; GALVÃO, V. da F. Estratégias de estudo em contexto universitário: implicações para a expertise. In: MONTEIRO, F. M. de A.; MULLER, M. L. R. (Org.). Educação como espaço da cultura. Cuiabá: Ed. da UFMT, 2006. v. 2, p. 221-238.

GALVÃO, A. C. T. Cognição, emoção e expertise musical. Psicologia: Teoria e Pesquisa, v. 22, n. 2, p. 169-174, 2006.

GALVÃO, A. C. T. A questão do talento: usos e abusos. In: VIRGOLIM, A. M. R. (Org.). Talento criativo: expressão em múltiplos contextos. Brasília: Editora UnB, 2007a. p. 121-142.

GALVÃO, A. C. T. Fatores associados ao desenvolvimento do talento musical. In: FLEITH, D.; ALENCAR, E. (Org.). Desenvolvimento de talentos e altas habilidades: orientação a pais e professores. Porto Alegre: Artmed, 2007b. p. 99-115. GALVÃO, A. C. T.; LACORTE, S. Processos de aprendizagem de músicos populares: um estudo exploratório. Revista da ABEM, Porto Alegre, v. 17, p. 29-38, 2007.

GOMES, A. C. A. A relação entre sujeito expert e objeto de conhecimento de expertise. 2008. 112 f. Dissertação (Mestrado em Educação) - Universidade Católica de Brasília, Brasília, 2008. 
HUNT, E. Expertise, talent, and social encouragement. In: ERICSSON, K. A. et al. (Ed.). The Cambridge handbook of expertise and expert performance. Cambridge; New York: Cambridge University Press, 2006. p. 31-38.

MAGILL, R. A. Aprendizagem motora: conceitos e aplicações. São Paulo: Edgard Blücher, 1984.

ORLANDI, E. de L. P. Análise do discurso: princípios e procedimentos. Campinas: Pontes, 2002.

RAVANELLO, J. Motivação para aprender: um estudo com universitários de Pedagogia e de Letras. 2008. 129 f. Dissertação (Mestrado em Educação) Universidade Católica de Brasília, Brasília, 2008.

SIMON, H.; CHASE, W. Skills in chess. American Scientist, v. 61, p. 394-403, 1973.

SOSNIAK, L. A. Learning to be a concert pianist. In: BLOOM, B. S. Developing talent in young people. New York: Ballantine Books, 1985. p. 19-67.

SOSNIAK, L. A. The tortoise, the hare, and the development of talent. In: HOWE, M. J. A. Encouraging the development of exceptional skills and talents. Leicester: British Psychological Society, 1990. p. 149-164.

THIEDE, K.; DUNLOSKY, J. Toward a general model of self-regulated study: an analysis of selection of items for study and self-paced study time. Journal of Experimental Psychology: Learning, Memory, and Cognition, v. 25, n. 4, p. 1024-1037, 1999.

WEINER, B. Achievement motivation. New Jersey: General Learning Press, 1974.

Recebido: 11/08/2010

Received: 08/11/2010

Aprovado: 09/12/2010

Approved: 12/09/2010 